\title{
Serum PON1 as a biomarker for the estimation of microvascular invasion in hepatocellular carcinoma
}

\author{
Guang-Yu Ding ${ }^{1,2 \#}$, Xiao-Dong Zhu ${ }^{1,2 \#}$, Yuan Ji ${ }^{3}$, Guo-Ming Shi ${ }^{1,2}$, Ying-Hao Shen ${ }^{1,2}$, Jian Zhou ${ }^{1,2}$, \\ Jia Fan ${ }^{1,2}$, Hui-Chuan Sun ${ }^{1,2}$, Cheng Huang ${ }^{1,2}$ \\ ${ }^{1}$ Department of Liver Surgery and Transplantation, Liver Cancer Institute, Zhongshan Hospital, Fudan University, Shanghai 200032, China; ${ }^{2}$ Key \\ Laboratory for Carcinogenesis and Cancer Invasion, the Chinese Ministry of Education, Shanghai 200032, China; ${ }^{3}$ Department of Pathology, \\ Zhongshan Hospital, Fudan University, Shanghai 200032, China \\ Contributions: (I) Conception and design: C Huang, HC Sun, J Fan; (II) Administrative support: J Fan, J Zhou; (III) Provision of study materials \\ or patients: GM Shi, YH Shen, J Zhou, HC Sun, C Huang; (IV) Collection and assembly of data: GY Ding, XD Zhu, Y Ji; (V) Data analysis and \\ interpretation: GY Ding, XD Zhu; (VI) Manuscript writing: All authors; (VII) Final approval of manuscript: All authors. \\ \#These authors contributed equally to this work. \\ Correspondence to: Cheng Huang. Department of Liver Surgery and Transplantation, Liver Cancer Institute, Zhongshan Hospital, Fudan University, \\ 136 Yi Xue Yuan Rd, Shanghai 200032, China. Email: huang.cheng@zs-hospital.sh.cn.
}

Background: Microvascular invasion (MVI) is considered as one of the most powerful prognostic factors in hepatocellular carcinoma (HCC). Currently, it could only be diagnosed by post-operative histological examination. This study aimed to assess the diagnostic value of serum paraoxonase 1 (PON1) for MVI.

Methods: In this study, we analyzed data from 754 HCC patients who underwent surgical treatment between December 2010 and November 2011. Serum PON1 was measured by ELISA and receiver operating characteristic (ROC) curve was applied to calculate diagnostic accuracy.

Results: MVI was detected in 174 of 505 patients (34.5\%) in the test cohort and 84 of 249 patients (33.7\%) in the validation cohort. Univariate analyses indicated tumor size, AFP, and PON1 were significantly related with vascular invasion status. ROC curves determined the optimum diagnostic cutoff value for PON1 was $191.12 \mathrm{ng} / \mathrm{mL}$ (AUC $0.754,95 \%$ CI: $0.710-0.798$, sensitivity $70.67 \%$, specificity $78.11 \%$ in the test cohort), which was significantly better than AFP (cutoff value $279.8 \mathrm{ng} / \mathrm{mL}$, AUC 0.666, 95\% CI: 0.618-0.714, sensitivity $40.38 \%$, specificity $85.19 \%, \mathrm{P}=0.0063$ ). In the sHCC sub-group, PON1 retained diagnostic value (AUC 0.738, 95\% CI: 0.680-0.796, sensitivity 72.82\%, specificity 76.57\% in the test cohort), while AFP failed to do so (AUC 0.579, 95\% CI: $0.511-0.647$, sensitivity $26.21 \%$, specificity $86.84 \%, \mathrm{P}=0.0003$ ). These results were further confirmed by the validation cohort. The combination of PON1 and AFP increased the diagnostic accuracy for vascular invasion compared with either test alone (AUC 0.785, 95\% CI: 0.744-0.826, sensitivity 75.96\%, specificity 77.44\%; PON1 plus AFP vs. PON1 alone, $\mathrm{P}=0.0004$; PON1 plus AFP vs. AFP alone, $\mathrm{P}<0.0001)$.

Conclusions: Serum PON1 could potentially be used to diagnose MVI and could be used to guide more personalized treatment strategy.

Keywords: Hepatocellular carcinoma (HCC); Paraoxonase 1; prediction; vascular invasion

Submitted Dec 17, 2019. Accepted for publication Jan 03, 2020.

doi: $10.21037 /$ atm.2020.01.44

View this article at: http://dx.doi.org/10.21037/atm.2020.01.44 


\section{Introduction}

Hepatocellular carcinoma (HCC) is the fifth most common malignancy and the third leading cause of cancer death worldwide (1). With the progression of surgical technology, curative resection and liver transplantation are now widely considered the first choice of therapy for HCC (2-4). Unfortunately, the high recurrence rate of HCC in the remnant liver remains a major obstacle for partial hepatectomy, and the need to select patients who will gain enduring benefit from surgical therapies is urgent (5-7). Microvascular invasion (MVI) is a powerful, validated, independent predictor of early recurrence and poor overall survival (OS) after surgical treatment for HCC $(8,9)$. Currently, MVI can only be detected by postresection histological examination, which greatly limits its clinical utility (10). A noninvasive test capable of accurately identifying MVI preoperatively would be of great benefit in better stratifying HCC patients for personalized management.

Paraoxonase 1 (PON1) is a calcium-dependent hydrolase protein synthesized mainly in the liver by hepatocytes and secreted into the circulatory system (11). Serum PON1 has been implicated in cell damage and chronic inflammation in the liver. In chronic liver disease, PON1 levels in serum and hepatic tissue correlate with the degree of liver damage (12). As an important anti-atherosclerotic factor, PON1 has the capacity to prevent oxidation of low-density lipoprotein (LDL), maintain the normal function of endothelial cells, inhibit the adhesion of leukocytes (especially monocytes and macrophage), and reduce chronic inflammation of the vascular wall (13-17). Because chronic inflammation and leukocyte adhesion are the hallmarks of tumor vascularization and abnormalities in tumor vessels play a crucial role in the process of tumor invasion and metastasis, we speculate that PON1 could play an important role in HCC vascular invasion. Through iTRAQ-based proteomic profiling and further confirmation, our previous study has revealed that serum PON1 was a potential diagnostic biomarker for MVI (18). This finding was also supported by another research team (19). However, these reports had limitations, such as small study size and absence of controls with non-malignant liver disease. Therefore, we designed a large-scale validation study to assess the diagnostic accuracy of PON1 as a preoperative, noninvasive, serum biomarker for MVI.

\section{Methods}

\section{Study population}

This study recruited consecutive patients who underwent surgical treatment at the Liver Cancer Institute, Zhongshan Hospital, Fudan University, Shanghai, China, from December 2010 to November 2011. The study population included patients with HCC, focal nodular hyperplasia (FNH), liver cyst, and hemangioma. All diagnosis was defined on the basis of ultrasound, CT, or MRI characteristics, and was confirmed by histopathology. No patients had a biopsy prior to the operation. Patients were excluded if they received preoperative treatments (i.e., ablation or transarterial chemoembolization).

HCC patients were classified based on their vascular invasion status: no vascular invasion (NVI) and vascular invasion (VI). The VI group was subdivided into MVI and gross vascular invasion (GVI). The diagnosis of vascular invasion was made on the basis of established criteria $(8,9)$. The presence of MVI was diagnosed when tumor cells were detected in microvessels on microscopic observations by two different pathologists. In most cases, the presence or absence of MVI was so obvious that the two reviewers had consistent results. Inconsistencies, if any, were resolved by discussion, as is the usual procedure for pathologic diagnosis in the hospital. The presence of GVI was diagnosed when tumor thrombi were observed in the first or second branches of the portal veins on postoperative pathologic observations or by preoperative radiological tests, such as CT or MRI. We also defined sHCC as tumor diameter less than $5 \mathrm{~cm}$ (including $5 \mathrm{~cm}$ ), and advanced-HCC as tumor diameter greater than $5 \mathrm{~cm}$.

Approval for the study was obtained from the ethics committee of Zhongshan hospital, Fudan University. Informed consent was obtained from participants, according to the committee's regulations.

\section{Testing of blood samples}

Peripheral blood samples were drawn within one week of CT or MRI diagnosis and prior to surgical treatment. Samples were centrifuged and stored at $-70 \mathrm{w}$ until testing. 
Assays for serum PON1 were completed by two researchers at Liver Cancer Institute, Shanghai, China, who had no access to patients' clinical information. A commercial ELISA kit (R\&D Systems, Minneapolis, MN, USA) was used according to the manufacturer's recommendations. Briefly, 96-well microplates were coated with $100 \mu \mathrm{L}$ of the monoclonal antibody to PON1 supplied with the ELISA kit $(1 \mu \mathrm{g} / \mathrm{mL})$ and incubated at $4{ }^{\circ} \mathrm{C}$ overnight. Nonspecific reactions were blocked with $1 \%$ bovine serum albumin. Patients' sera diluted with $10 \%$ neonatal calf serum were incubated for $2 \mathrm{~h}$ at $37^{\circ} \mathrm{C}$. Incubation with the detection antibody, biotinylated goat antihuman PON1 $(600 \mathrm{ng} / \mathrm{mL})$, was performed for $2 \mathrm{~h}$ at $37^{\circ} \mathrm{C}$, followed by the addition of $100 \mu \mathrm{L}$ of a 1:200 dilution of streptavidin horseradish peroxidase for $20 \mathrm{~min}$. Color development was achieved with $100 \mu \mathrm{L}$ per well of 3,3,5,5-tetramethylbenzidine and hydrogen peroxide as a substrate, and sulfuric acid $(1 \mathrm{~mol} / \mathrm{L})$ was added to stop the reaction. The optical density was measured at $450 \mathrm{~nm}$ and referenced to $570 \mathrm{~nm}$ on a Synergy 2 multimode plate reader (Biotek, Winooski, VT, USA). The concentrations of PON1 were obtained with a fourparameter logistic curve, fit to the standard value and multiplied by the dilution factor. All measurements were performed in duplicate.

\section{Statistical analysis}

Statistical analyses were done with SPSS 20.0 software (IBM, Armonk, NY, USA). Differences between two independent groups were tested with the Mann-Whitney U test (continuous variables and nonparametric analyses). Receiver operating characteristics (ROC) curves were constructed to assess sensitivity, specificity, and respective areas under the curves (AUCs) with 95\% confidence interval (CI). Differences between AUCs were tested following the method of DeLong et al. We investigated the optimum cutoff value for diagnosis by maximizing the sum of sensitivity and specificity and minimizing the overall error [square root of the sum $(1-\text { sensitivity })^{2}+(1-\text { specificity })^{2}$ ], and by minimizing the distance of the cutoff value to the top-left corner of the ROC curve. To test the diagnostic accuracy when both PON1 and $\alpha$-fetoprotein (AFP) were measured, we estimated functions of the combined marker by binary logistic regression, and the values of these functions were used as one marker and subjected to ROC analysis. The correlation between PON1 concentrations in serum and clinicopathological characteristics was analyzed with Pearson's $\chi^{2}$ test or Fisher's exact test. To assess whether the combined use of PON1 and AFP measurement was better than either of these two biomarkers alone, a new variable predicted probability $(\mathrm{P})$ for $\mathrm{VI}$ was created on the basis of an equation obtained by binary logistic regression (VI group versus NVI group in the test cohort):

$$
\ln (\mathrm{P} / 1-\mathrm{P})=0.620994-0.004311 \times \mathrm{PON} 1+0.000106 \times
$$
AFP

We took $\mathrm{P}$ values lower than 0.05 (two sided) to be significant.

\section{Results}

\section{Patient characteristics}

For 861 patients screened, 505 HCC patients were recruited into the test cohort from December 2010 to July 2011, and 249 HCC patients were recruited into the validation cohort from August 2011 to November 2011. The remaining 107 patients were diagnosed with benign liver disease that consisted of 21 FNHs, 25 cysts, and 61 hemangiomas (Figure 1). Clinicopathological characteristics of HCC patients in the test and validation cohorts are summarized in Table S1. The cohorts were well matched for most factors. The majority of the study population were HBV-related HCCs $(86.7 \%$ in the test cohort, $80.3 \%$ in the validation cohort). In contrast, only $13.3 \%$ patients in the test cohort were infected with $\mathrm{HCV}$ while no $\mathrm{HCV}$ patient was enrolled in the validation cohort. The vascular invasion status of the HCC patients is also illustrated in Table 1, MVI and GVI were detected in $34.5 \%$ and $6.7 \%$ patients, respectively, in the test cohort, which was slightly different from the validation cohort (33.7\% with MVI and $12.0 \%$ with GVI, $\mathrm{P}=0.045$ ).

Univariate analyses were adopted to identify possible preoperative characteristics for VI, and only tumor size, $\mathrm{AFP}$ and PON1 were significantly related with VI (Table 1, $\mathrm{P}<0.001)$.

\section{PON1 and AFP serum levels in different group}

PON1 concentrations on ELISA of different liver diseases are illustrated in Figure S1. Although we could notice a trend of decrease in PON1 concentration in HCC compared to benign liver diseases, the differences between HCC and FNH or Cyst patients failed to reach statistical significance (both $\mathrm{P}>0.05$ ), but the PON1 concentration was significantly higher in hemangioma patients than in HCC $(\mathrm{P}<0.05)$. 


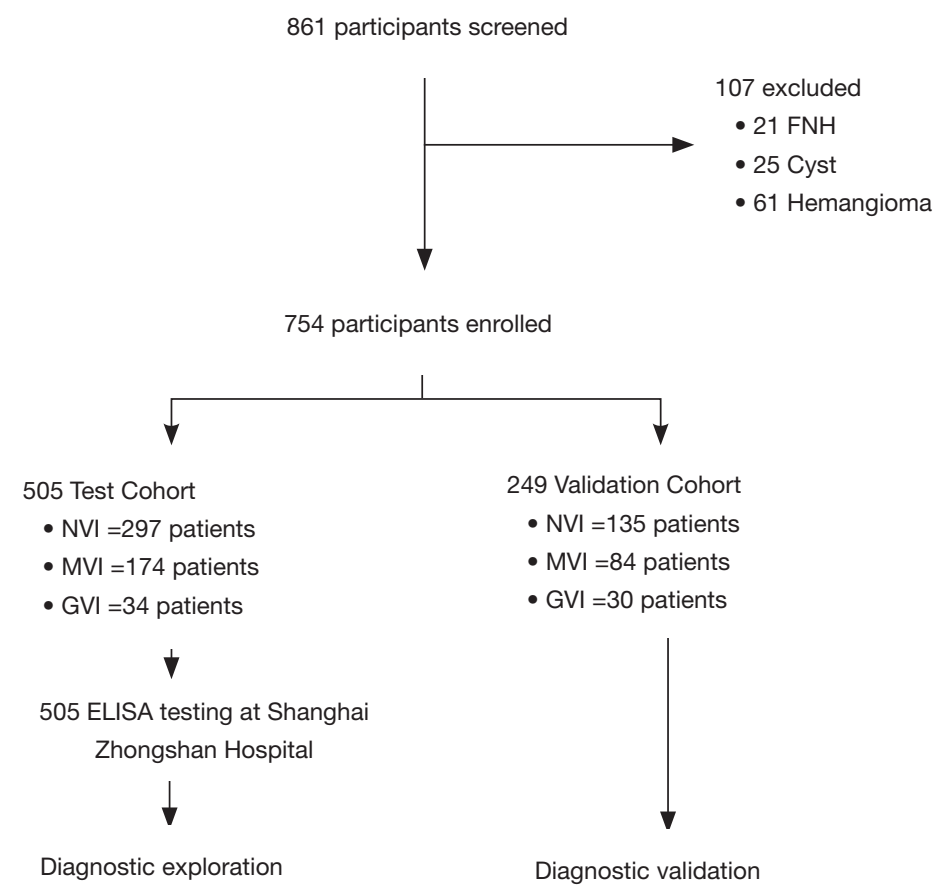

Figure 1 Study profile. FNH, focal nodular hyperplasia; NVI, no vascular invasion; MVI, micro-vascular invasion; GVI, gross vascular invasion; ELISA, enzyme-linked immunosorbent assay.

The serum PON1 and AFP concentrations in HCC patients with different VI status are further demonstrated in Figure 2 and Table S2. PON1 concentration was significantly related with vascular invasion status both in the test and validation cohort (Figure 2A,B). Patients without vascular invasion had significantly higher level of PON1 than patients with MVI $(\mathrm{P}<0.0001$ in test cohort, $\mathrm{P}=0.0036$ in validation cohort), and patients with GVI had the lowest PON1 level even compared to the MVI subgroup $(\mathrm{P}<0.05$ in both cohorts). An inversed correlation between AFP concentration and vascular invasion was also noticed (Figure 2C,D, Table S2).

\section{Performance of PON1, AFP and combination in predicting vascular invasion status}

ROC curves showed the optimum diagnostic cutoff value for PON1 was $191.12 \mathrm{ng} / \mathrm{mL}$ (AUC 0.754, 95\% CI: 0.710-0.798, sensitivity $70.67 \%$, specificity $78.11 \%$; Figure 3, Table 2). The optimum cutoff value for AFP was $279.8 \mathrm{ng} / \mathrm{mL}$ (AUC 0.666, 95\% CI: 0.618-0.714, sensitivity 40.38\%, specificity $85.19 \%$; Figure 3, Table 2). The cutoff values adopted in this study were based on VI versus NVI group in the test cohort, but we acquired the same result when we excluded the GVI patients from the ROC curves. Predictive values and likelihood ratios for PON1 and AFP in the diagnosis of vascular invasion are shown in Table 2.

In the assessment of diagnostic accuracy, serum PON1 had greater AUC and sensitivity values than did AFP in the test cohort $(\mathrm{P}=0.0063)$, and specificity was better with AFP in the test cohort while the validation cohort indicated different result (Figure 3, Table 2). We also noticed decreased serum PON1 could still effectively distinguish patients with MVI from those with NVI when we ruled out GVI patients (AUC 0.737 , sensitivity $68.39 \%$, specificity $78.11 \%$ ) which was also significantly better than AFP (Figure 3, Table 2, $\mathrm{P}=0.0047$ ).

Tumor size was an important factor of VI, $31.1 \%$ patients with sHCC had VI while the rate significantly increased to $60.3 \%$ in patients with advanced-HCC (Table 1). In the test cohort, $331(65.5 \%)$ of 505 patients had sHCC, and the AUC of PON1 was 0.738 (95\% CI: 0.680-0.796) with sensitivity of $72.82 \%$ and specificity of $76.57 \%$ significantly better than those of AFP (AUC $0.579,95 \%$ CI: $0.511-0.647$, sensitivity $26.21 \%$, specificity $86.84 \%$, Table 2, Figure 4, $\mathrm{P}=0.0003)$. The diagnostic power of $\mathrm{PON} 1$ 
Table 1 Univariate analyses of factors associated with vascular invasion in the test cohort

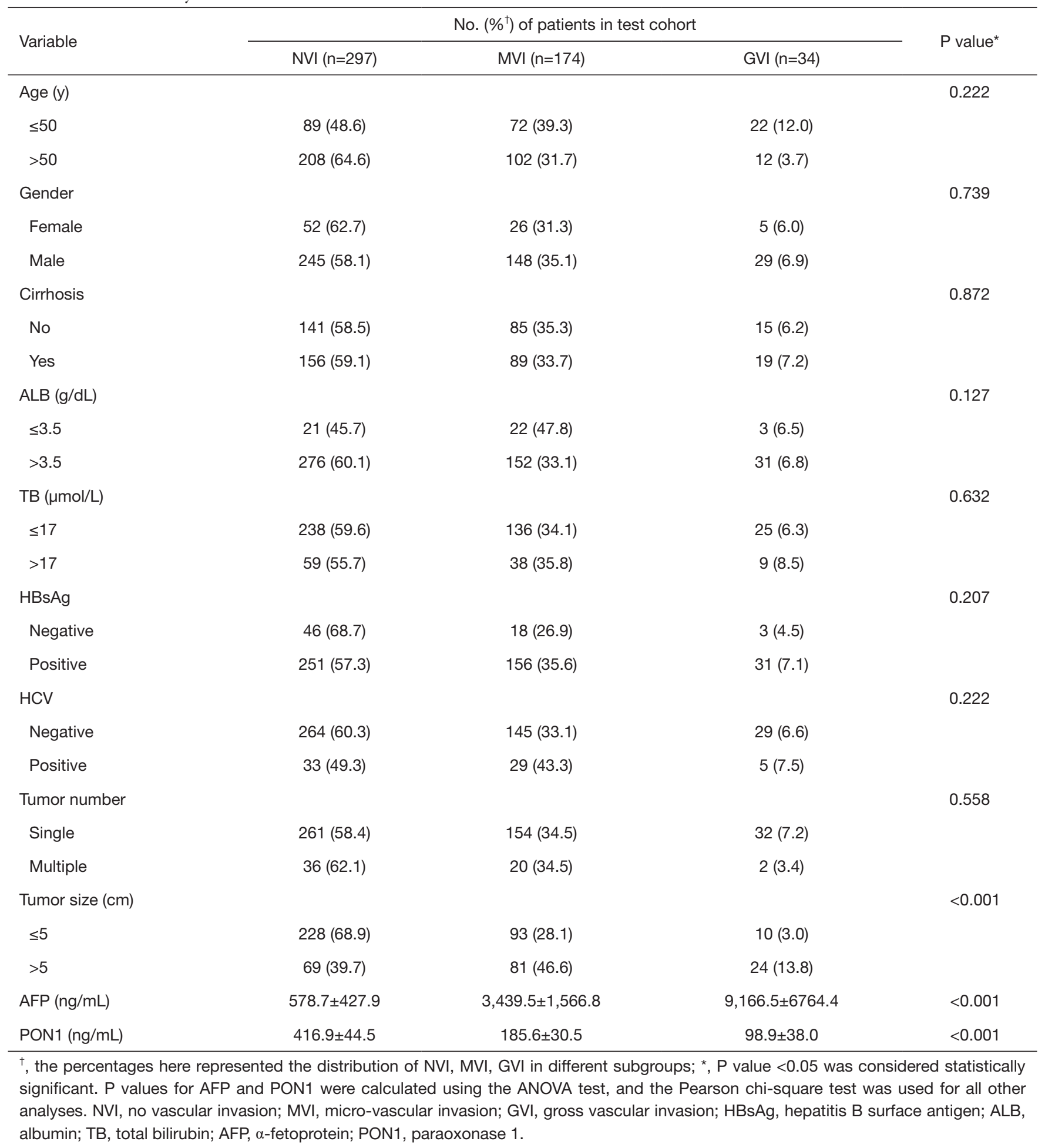




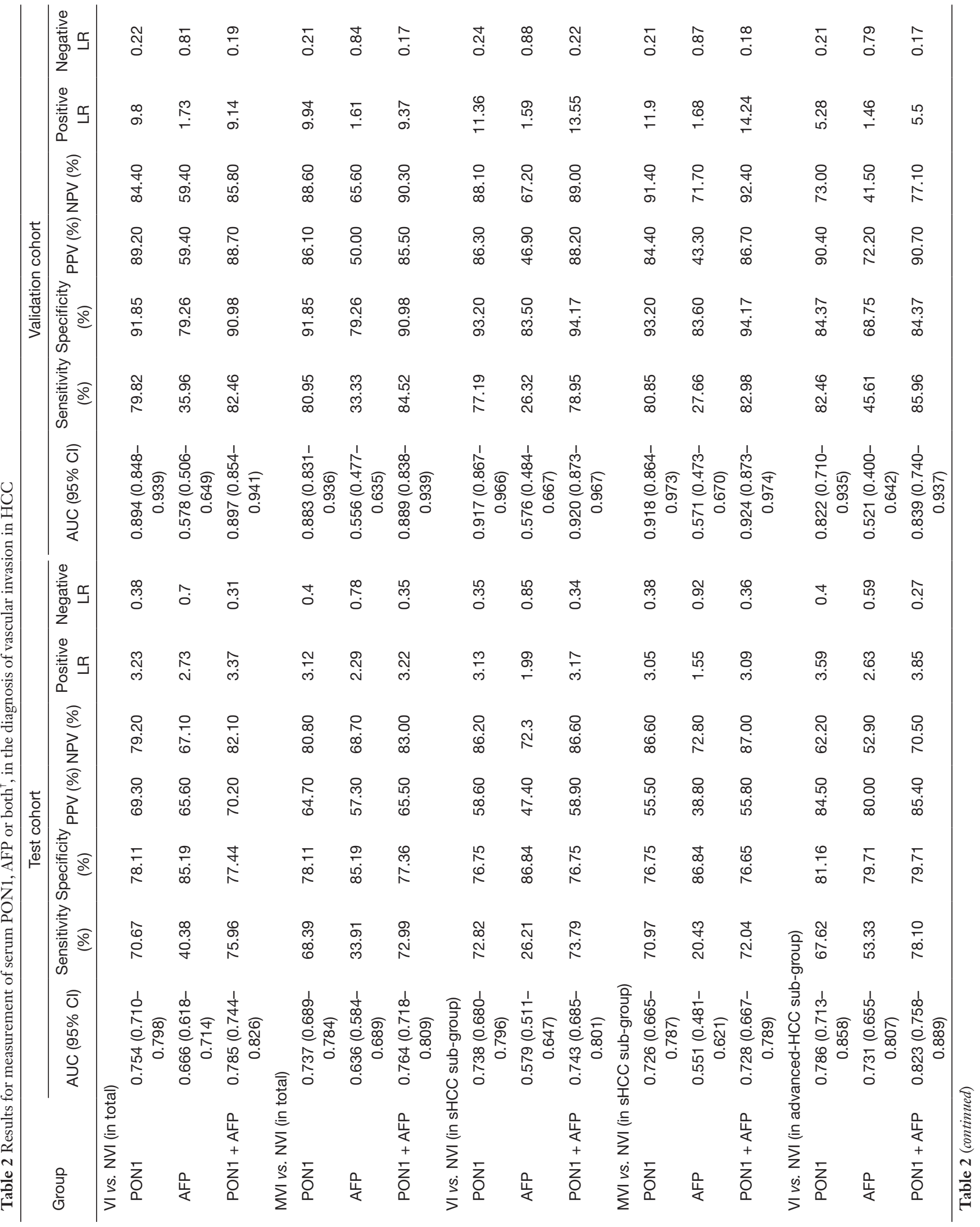




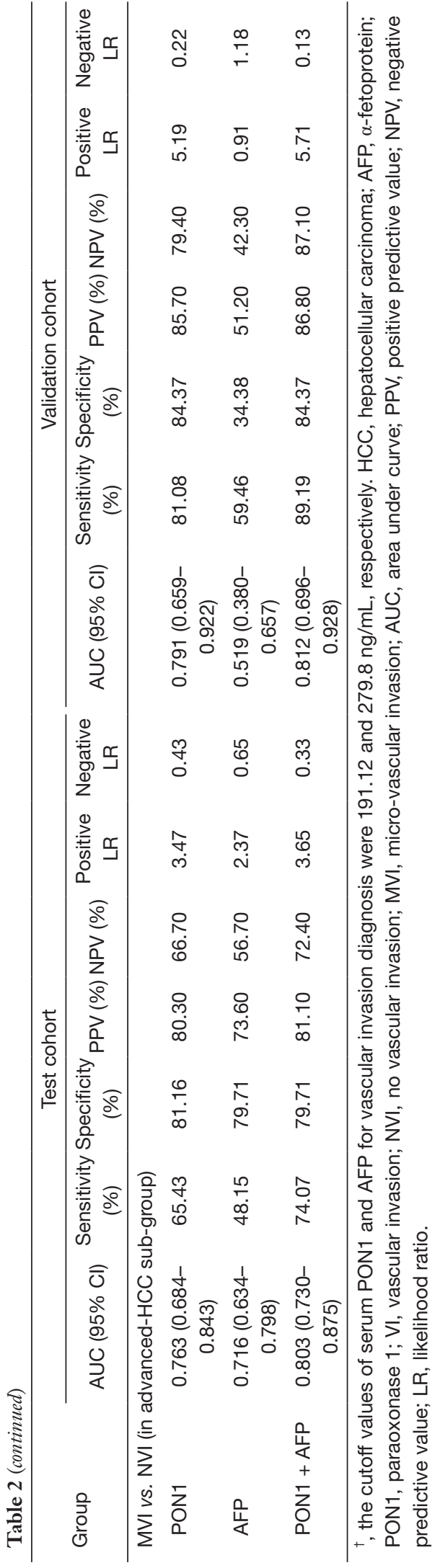

in the advanced-HCC sub-group remained valuable (AUC 0.786, 95\% CI: $0.713-0.858$, sensitivity $67.62 \%$, specificity $81.16 \%$, Table 2, Figure 5). Compared to AFP, the ROC curves for PON1 indicated a diagnosis of VI irrespective of tumor size (Table 2, Figures 4,5).

ROC analysis showed that testing of both PON1 and AFP increased the diagnostic accuracy for VI compared to either test alone (AUC 0.785, 95\% CI: 0.744-0.826, sensitivity $75.96 \%$, specificity $77.44 \%$; PON1 plus AFP $v$ s. PON1 alone, $\mathrm{P}=0.0004 ; \mathrm{PON} 1$ plus AFP vs. AFP alone, $\mathrm{P}<0.0001$; Table 2, Figure 3). Diagnostic accuracy of the combination of PON1 and AFP remained improved when only the advanced-HCC sub-group was assessed (AUC 0.823, 95\% CI: $0.758-0.889$, sensitivity $78.10 \%$, specificity $79.71 \%$; Figure 5, Table 2). However, in the sHCC subgroup, this combination did not demonstrate better results compared to PON1 alone (Figure 4, Table 2, $\mathrm{P}=0.1851$ ).

With use of the $191.12 \mathrm{ng} / \mathrm{mL}$ threshold for PON1 and $279.8 \mathrm{ng} / \mathrm{mL}$ for AFP, we observed similar or better results in the validation cohort to those in the test cohort. PON1 had good diagnostic accuracy of VI, especially MVI, for HCC (Table 2, Figure 3). Additionally, the validation cohort confirmed the ability of PON1 to diagnose VI in sHCC sub-group when AFP failed to do so (Figure 4, Table 2). The improvement in diagnostic accuracy for VI by measurement of PON1 and AFP together was also proven in the validation cohort (Figure 3, Table 2). The detailed data of the ROC curves was demonstrated in Table S3.

\section{Discussion}

High rates of recurrence are the major causes of the poor prognosis of HCC after surgery $(20,21)$. Current management guidelines do not account for histological MVI, a well-known predictor of recurrence and poor outcomes for HCC $(9,22-24)$. As the two main categories of VI, GVI can be diagnosed preoperatively using radiological examination, while the detection of MVI is achieved only by histological observations in resected tissues $(25,26)$. In previous studies, AFP combined with tumor size or other methods have been identified as predictors of MVI preoperatively, but the effectiveness or convenience was far from satisfactory (27-29). A previous study identified PIVKA-II to be a better biomarker of MVI than AFP with an AUC of 0.81 , but the study population was limited to 85 HCC patients (30). Other studies tried to solve this problem by using different scoring systems or nomograms which integrated several clinical factors, but all these findings 

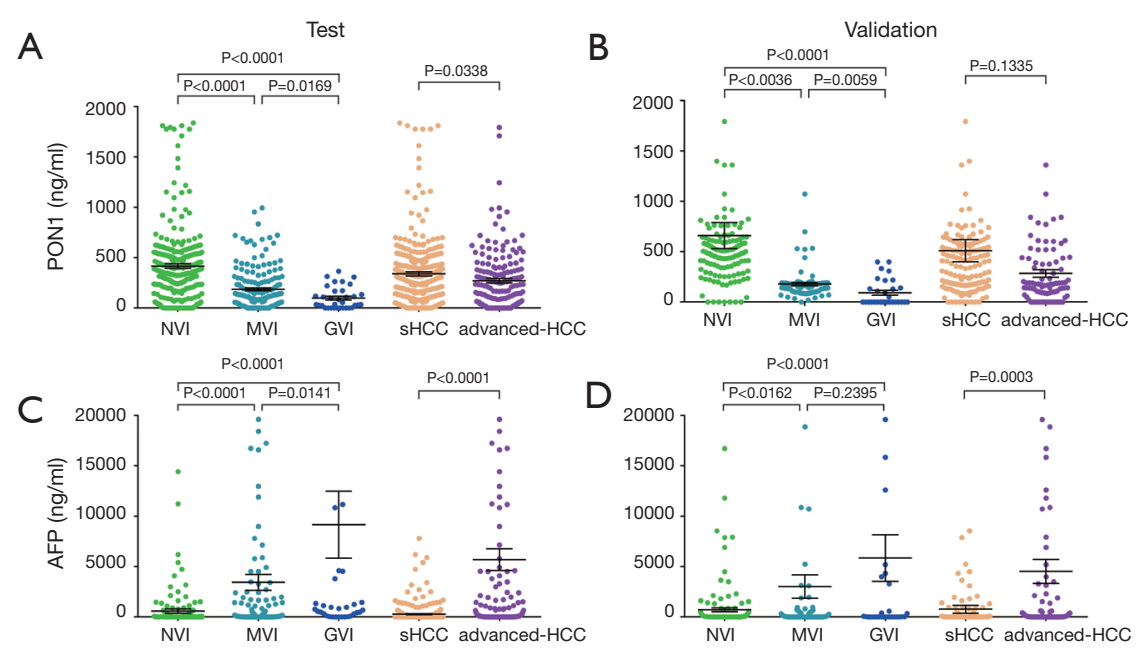

Figure 2 PON1 and AFP concentrations in serum in the test and validation cohorts. (A) PON1 for test cohort; (B) PON1 for validation cohort; (C) AFP for test cohort; (D) AFP for validation cohort. Black horizontal lines are means, and error bars are SEs. 2,000 ng/mL was used as the upper limit for PON1, and 20,000 ng/mL was used as the upper limit for AFP. NVI, no vascular invasion; MVI, micro-vascular invasion; GVI, gross vascular invasion; AFP, $\alpha$-fetoprotein; PON1, paraoxonase 1.
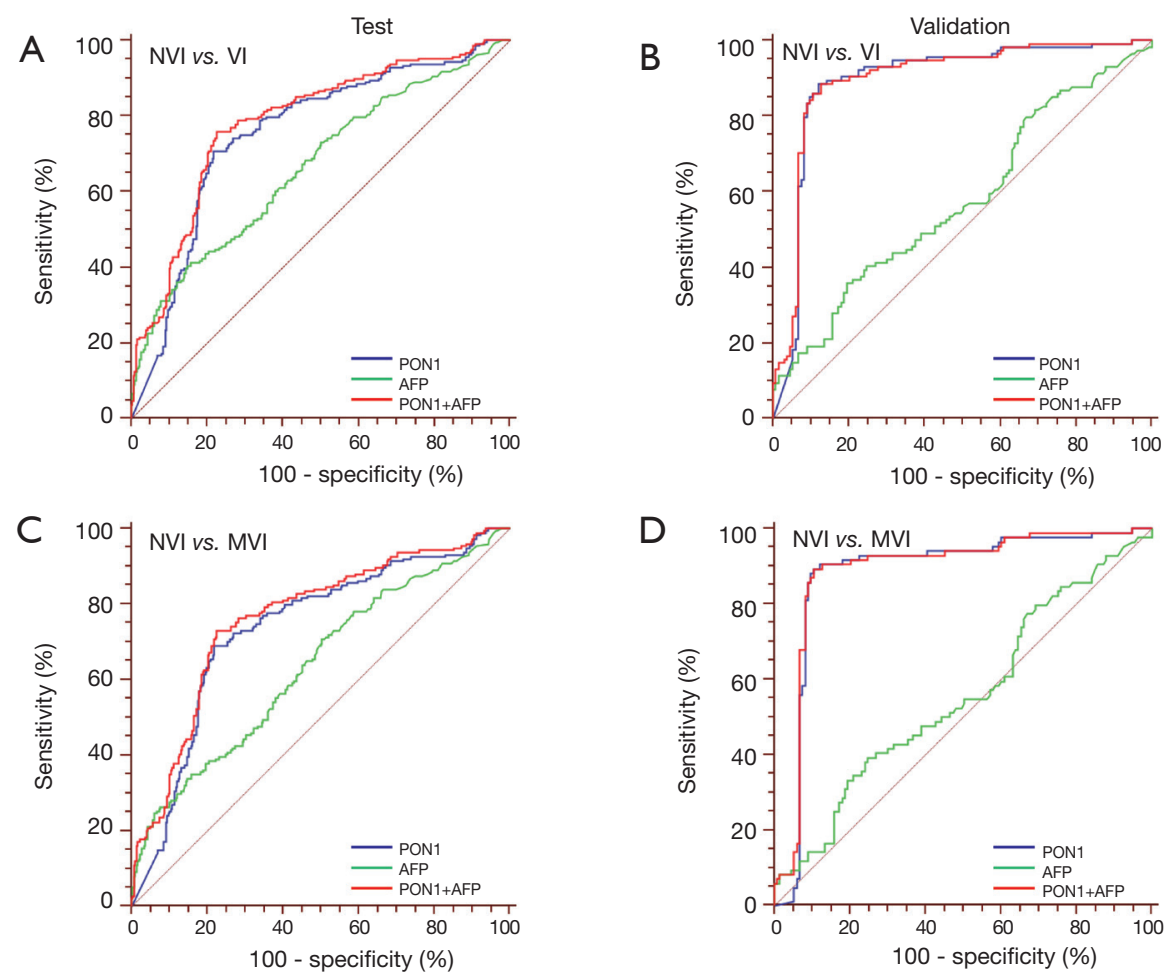

Figure 3 Diagnostic outcomes for serum PON1 in the diagnosis of vascular invasion of HCC. (A) ROC curve for PON1, AFP, or both for all patients with NVI versus VI in the test cohort; (B) ROC curve for PON1, AFP, or both for all patients with NVI versus VI in the validation cohort; (C) ROC curve for PON1, AFP, or both for all patients with NVI versus MVI in the test cohort; (D) ROC curve for PON1, AFP, or both for all patients with NVI versus MVI in the validation cohort. AFP, $\alpha$-fetoprotein; PON1, paraoxonase 1; VI, vascular invasion; NVI, no vascular invasion; MVI, micro-vascular invasion; ROC, receiver operating characteristics. 

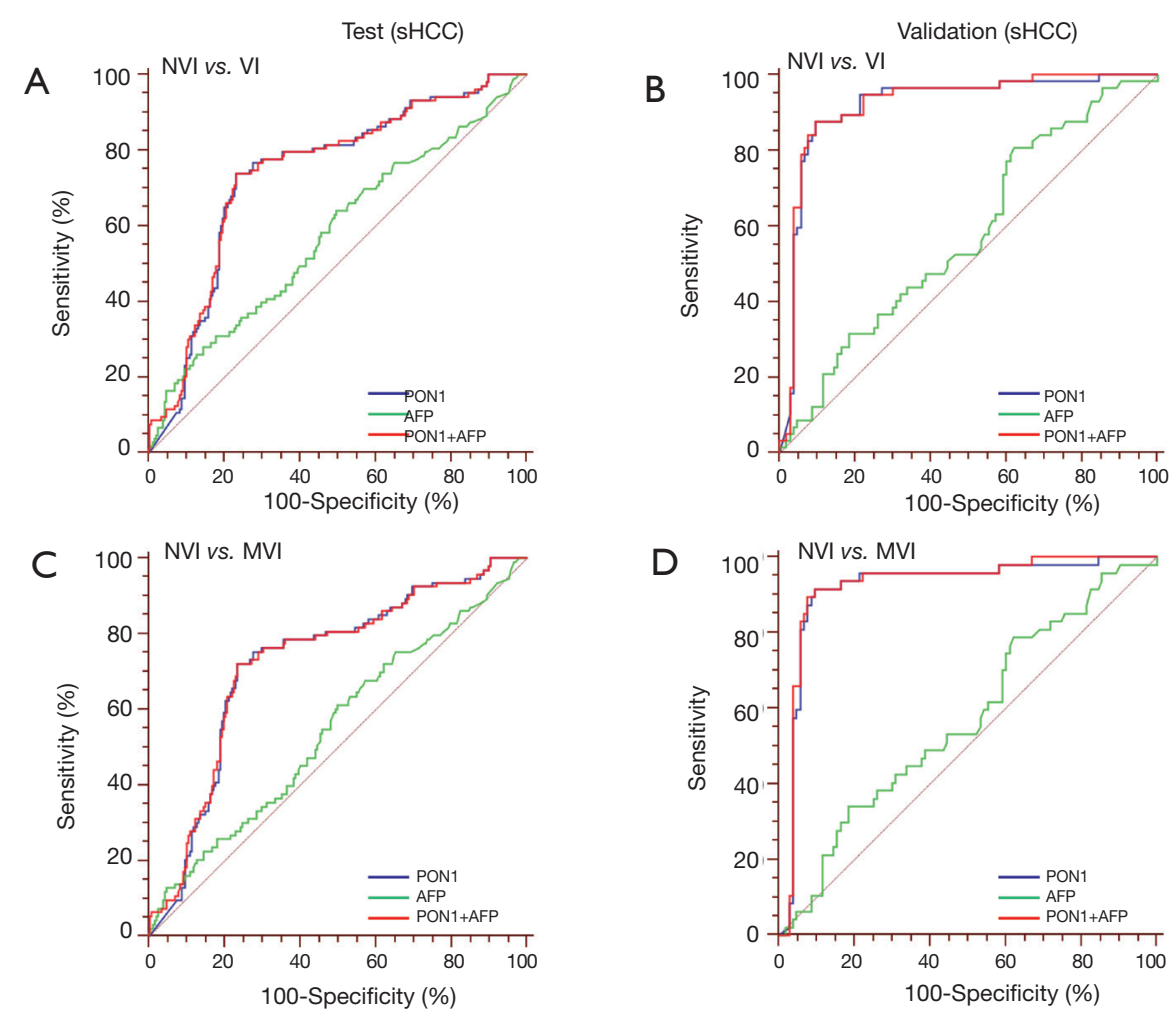

Figure 4 Diagnostic outcomes for serum PON1 in the diagnosis of vascular invasion of sHCC. (A) ROC curve for PON1, AFP, or both for sHCC patients with NVI versus VI in the test cohort; (B) ROC curve for PON1, AFP, or both for sHCC patients with NVI versus VI in the validation cohort; (C) ROC curve for PON1, AFP, or both for sHCC patients with NVI versus MVI in the test cohort; (D) ROC curve for PON1, AFP, or both for sHCC patients with NVI versus MVI in the validation cohort. AFP, $\alpha$-fetoprotein; PON1, paraoxonase 1; VI, vascular invasion; NVI, no vascular invasion; MVI, micro-vascular invasion; ROC, receiver operating characteristics.

were not validated by large-scale study and were not ready for clinical application. In this study, we have shown that measurement of serum PON1 has a diagnostic value for MVI better than that of AFP, especially for patients with sHCC. The AUC of PON1 to diagnose VI was 0.754 (95\% CI: $0.710-0.798)$ in HCC patients, and 0.738 (95\% CI: $0.680-0.796)$ in the sHCC subgroup, both significantly higher than AFP $(\mathrm{P}<0.01)$.

PON1 is a calcium-dependent hydrolase protein synthesized mainly in the liver and secreted into the circulatory system (13), and generally its level in serum correlates with the degree of cell damage and chronic inflammation in the liver (12). However, the immunohistochemical study from our previous work demonstrated that PON1 expression in tumor tissues was inversely correlated with vascular invasion status, whereas its expression in peri-tumor tissues showed no significant association (18). This finding suggested that the serum concentration of PON1 in HCC patients might be modulated primarily by PON1 expression in the tumor tissue rather than non-tumoral hepatic tissues, and the decreased level of serum PON1 might reflect the progression of HCC. As an important antiatherosclerotic factor, PON1 involves in the cellular cholesterol synthesis, it has the capacity to maintain the normal function of endothelial cells, inhibit the adhesion of leukocytes (especially monocytes and macrophages), and reduce chronic inflammation of the vascular wall (14). As disrupted cholesterol homeostasis, chronic inflammation and leukocyte adhesion are the hallmarks of tumor vascularization and could lead to tumor invasion/metastasis, the anti-inflammatory and vessel normalization roles of PON1 could underlie the inverse correlation between serum PON1 level and vascular invasion $(16,17,31,32)$.

Compared to advanced HCC, the identification of VI in sHCC is more difficult and important in clinical practice. 

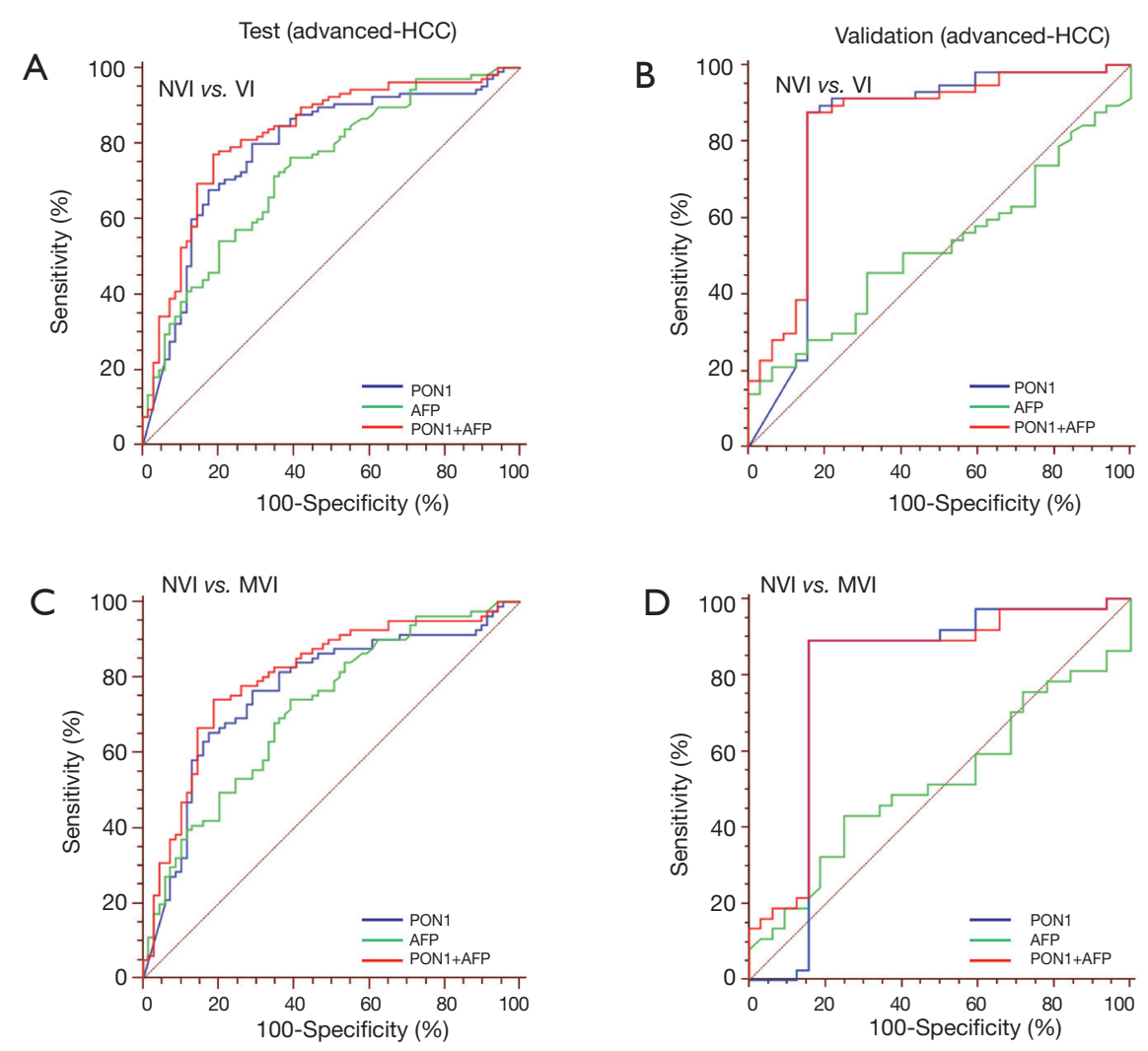

Figure 5 Diagnostic outcomes for serum PON1 in the diagnosis of vascular invasion of advanced-HCC. (A) ROC curve for PON1, AFP, or both for advanced-HCC patients with NVI versus VI in the test cohort; (B) ROC curve for PON1, AFP, or both for advanced-HCC patients with NVI versus VI in the validation cohort; (C) ROC curve for PON1, AFP, or both for advanced-HCC patients with NVI versus MVI in the test cohort; (D) ROC curve for PON1, AFP, or both for advanced-HCC patients with NVI versus MVI in the validation cohort. AFP, $\alpha$-fetoprotein; PON1, paraoxonase 1; VI, vascular invasion; NVI, no vascular invasion; MVI, micro-vascular invasion; ROC, receiver operating characteristics.

Our results demonstrated that, unlike AFP, the diagnostic power of PON1 did not significantly vary with tumor size. The difference of PON1 level between sHCC and advanced HCC was not as significant as that of AFP. In the advanced-HCC sub-group, AFP could achieve an AUC of 0.731 , while it dramatically dropped to 0.579 in the sHCC sub-group. However, the AUC of PON1 in sHCC and advanced HCC was 0.738 and 0.786 , respectively. This result confirmed that PON1 was an independent indicator of VI from tumor size.

Although the combination of PON1 and AFP increased the diagnostic accuracy for VI in total patients, it did not demonstrate better results compared to PON1 alone in the sHCC sub-group. Considering the convenience for clinical application, we suppose that PON1 alone might be sufficient.
The sample size and several clinical characteristics in our validation cohort were smaller and different from the test cohort. Therefore, the results of diagnostic performance differed between the two cohorts. Generally speaking, the prediction power of $\mathrm{PON} 1$ was even better in the validation cohort than in test cohort. For instance, the AUC of PON1 in all HCC patients was 0.894 in the validation cohort and 0.754 in the test cohort; the AUC of PON1 in the sHCC subgroup was 0.917 in the validation cohort and 0.738 in the test cohort. We also noticed that no HCV patients were recruited into the validation cohort, which was significantly different from the test cohort (which included $13.3 \% \mathrm{HCV}$ patients, $\mathrm{P}<0.001)$. This might be the explanation for the better diagnostic power of PON1 in the validation cohort, and the application of PON1 in HCV-related patients might not be as accurate as in $\mathrm{HBV}$-related patients. 
Interestingly, we observed an inverse result with AFP: the predictive accuracy was worse in the validation cohort than in the test cohort. The underlying mechanisms require further investigation.

There are some limitations of this study. First, our study is a single center, cross-sectional and retrospective study. Second, the majority of the study population were HBVrelated, and whether PON1 could be applied in HCVrelated patients remains doubtful. Therefore, we plan to do a prospective multicenter study to assess whether the use of PON1 can be validated in patients with or without $\mathrm{HBV}$ infection.

To our knowledge, this is the first large-scale study to report the clinically diagnostic relevance of PON1 as a serum protein marker for vascular invasion in HCC patients. Our results indicate that serum PON1 could potentially be used to diagnose VI, especially MVI, before surgery, and could be used to guide more personalized treatment strategy.

\section{Acknowledgments}

Funding: This work was supported by the National Natural Science Foundation of China (grant numbers 81502006 , 81572298 and 81871929).

\section{Footnote}

Conflicts of Interest: The authors have no conflicts of interest to declare.

Ethical Statement: The authors are accountable for all aspects of the work in ensuring that questions related to the accuracy or integrity of any part of the work are appropriately investigated and resolved. This study was approved by the Zhongshan Hospital Fudan University Research Ethics Committee. Informed consent was obtained according to the regulations. Informed consent obtained from the patients was written.

Open Access Statement: This is an Open Access article distributed in accordance with the Creative Commons Attribution-NonCommercial-NoDerivs 4.0 International License (CC BY-NC-ND 4.0), which permits the noncommercial replication and distribution of the article with the strict proviso that no changes or edits are made and the original work is properly cited (including links to both the formal publication through the relevant DOI and the license).
See: https://creativecommons.org/licenses/by-nc-nd/4.0/.

\section{References}

1. Siegel RL, Miller KD, Jemal A. Cancer Statistics, 2017. CA Cancer J Clin 2017;67:7-30.

2. Bruix J, Reig $M$, Sherman M. Evidence-based diagnosis, staging, and treatment of patients with hepatocellular carcinoma. Gastroenterology 2016;150:835-53.

3. Heimbach JK, Kulik LM, Finn RS, et al. AASLD guidelines for the treatment of hepatocellular carcinoma. Hepatology 2018;67:358-80.

4. Bruix J, Llovet JM. Major achievements in hepatocellular carcinoma. Lancet 2009;373:614-6.

5. Kolly P, Waidmann O, Vermehren J, et al. Hepatocellular carcinoma recurrence after direct antiviral agent treatment: A European multicentre study. J Hepatol 2017;67:876-8.

6. Gerbes A, Zoulim F, Tilg H, et al. Gut roundtable meeting paper: selected recent advances in hepatocellular carcinoma. Gut 2018;67:380-8.

7. Zheng J, Chou JF, Gonen M, et al. Prediction of Hepatocellular Carcinoma Recurrence Beyond Milan Criteria After Resection: Validation of a Clinical Risk Score in an International Cohort. Ann Surg 2017;266:693-701.

8. Roayaie S, Blume IN, Thung SN, et al. A system of classifying microvascular invasion to predict outcome after resection in patients with hepatocellular carcinoma. Gastroenterology 2009;137:850-5.

9. Lim KC, Chow PK, Allen JC, et al. Microvascular invasion is a better predictor of tumor recurrence and overall survival following surgical resection for hepatocellular carcinoma compared to the Milan criteria. Ann Surg 2011;254:108-13.

10. Rodríguez-Perálvarez M, Luong TV, Andreana L, et al. A systematic review of microvascular invasion in hepatocellular carcinoma: diagnostic and prognostic variability. Ann Surg Oncol 2013;20:325-39.

11. Mackness M, Mackness B. Human paraoxonase-1 (PON1): Gene structure and expression, promiscuous activities and multiple physiological roles. Gene 2015;567:12-21.

12. Marsillach J, Camps J, Ferre N, et al. Paraoxonase-1 is related to inflammation, fibrosis and PPAR delta in experimental liver disease. BMC Gastroenterol 2009;9:3.

13. Précourt LP, Amre D, Denis MC, et al. The threegene paraoxonase family: physiologic roles, actions and regulation. Atherosclerosis 2011;214:20-36.

14. Bouman HJ, Schomig E, van Werkum JW, et al. 
Page 12 of 12

Paraoxonase-1 is a major determinant of clopidogrel efficacy. Nat Med 2011;17:110-6.

15. Wheeler JG, Keavney BD, Watkins H, et al. Four paraoxonase gene polymorphisms in 11212 cases of coronary heart disease and 12786 controls: meta-analysis of 43 studies. Lancet 2004;363:689-95.

16. Coussens LM, Werb Z. Inflammation and cancer. Nature 2002;420:860-7.

17. Carmeliet P, Jain RK. Principles and mechanisms of vessel normalization for cancer and other angiogenic diseases. Nat Rev Drug Discov 2011;10:417-27.

18. Huang C, Wang Y, Liu S, et al. Quantitative proteomic analysis identified paraoxonase 1 as a novel serum biomarker for microvascular invasion in hepatocellular carcinoma. J Proteome Res 2013;12:1838-46.

19. Mínguez B, Hoshida Y, Villanueva A, et al. Geneexpression signature of vascular invasion in hepatocellular carcinoma. J Hepatol 2011;55:1325-31.

20. Forner A, Llovet JM, Bruix J. Hepatocellular carcinoma. Lancet 2012;379:1245-55.

21. Zhou J, Sun HC, Wang Z, et al. Guidelines for Diagnosis and Treatment of Primary Liver Cancer in China (2017 Edition). Liver Cancer 2018;7:235-60.

22. Tsai TJ, Chau GY, Lui WY, et al. Clinical significance of microscopic tumor venous invasion in patients with resectable hepatocellular carcinoma. Surgery 2000;127:603-8.

23. Duffy JP, Vardanian A, Benjamin E, et al. Liver transplantation criteria for hepatocellular carcinoma should be expanded: a 22-year experience with 467 patients at UCLA. Ann Surg 2007;246:502-9; discussion 509-11.

24. Yamashita Y, Shirabe K, Aishima S, et al. Predictors of

Cite this article as: Ding GY, Zhu XD, Ji Y, Shi GM, Shen YH, Zhou J, Fan J, Sun HC, Huang C. Serum PON1 as a biomarker for the estimation of microvascular invasion in hepatocellular carcinoma. Ann Transl Med 2020;8(5):204. doi: 10.21037/atm.2020.01.44

\section{Ding et al. PON1 as a serum biomarker for the prediction of MVI}

Microvascular Invasion in Hepatocellular Carcinoma. Dig Dis 2015;33:655-60.

25. Shirabe K, Itoh S, Yoshizumi T, et al. The predictors of microvascular invasion in candidates for liver transplantation with hepatocellular carcinoma-with special reference to the serum levels of des-gamma-carboxy prothrombin. J Surg Oncol 2007;95:235-40.

26. Lei Z, Li J, Wu D, et al. Nomogram for Preoperative Estimation of Microvascular Invasion Risk in Hepatitis B Virus-Related Hepatocellular Carcinoma Within the Milan Criteria. JAMA Surg 2016;151:356-63.

27. McHugh PP, Gilbert J, Vera S, et al. Alpha-fetoprotein and tumour size are associated with microvascular invasion in explanted livers of patients undergoing transplantation with hepatocellular carcinoma. HPB (Oxford) 2010;12:56-61.

28. Sumie S, Kuromatsu R, Okuda K, et al. Microvascular invasion in patients with hepatocellular carcinoma and its predictable clinicopathological factors. Ann Surg Oncol 2008; 15:1375-82.

29. Banerjee S, Wang DS, Kim HJ, et al. A computed tomography radiogenomic biomarker predicts microvascular invasion and clinical outcomes in hepatocellular carcinoma. Hepatology 2015;62:792-800.

30. Poté N, Cauchy F, Albuquerque M, et al. Performance of PIVKA-II for early hepatocellular carcinoma diagnosis and prediction of microvascular invasion. J Hepatol 2015;62:848-54.

31. Hanahan D, Weinberg RA. Hallmarks of cancer: the next generation. Cell 2011;144:646-74.

32. Jiang Y, Sun A, Zhao Y, et al. Proteomics identifies new therapeutic targets of early-stage hepatocellular carcinoma. Nature 2019;567:257-61. 


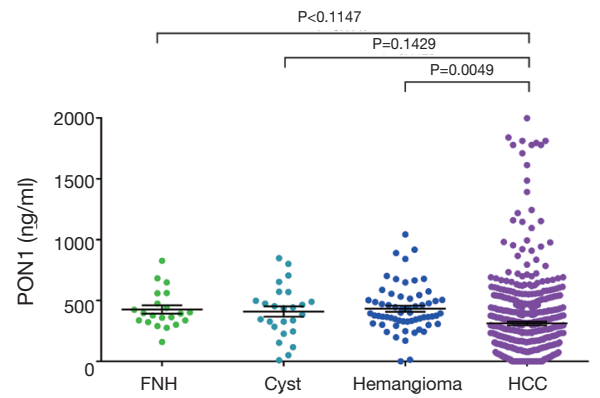

Figure S1 PON1 concentrations on ELISA of different liver diseases.

Table S1 The clinicopathologic characteristics of HCC patients in the test and validation cohorts

\begin{tabular}{|c|c|c|c|c|c|}
\hline \multirow{2}{*}{ Variable } & \multicolumn{2}{|c|}{ Test $(n=505)$} & \multicolumn{2}{|c|}{ Validation $(n=249)$} & \multirow{2}{*}{$P$ value } \\
\hline & No. & $\%$ & No. & $\%$ & \\
\hline \multicolumn{6}{|l|}{ Age (y) } \\
\hline$\leq 50$ & 183 & 36.2 & 106 & 42.6 & 0.093 \\
\hline$>50$ & 322 & 63.8 & 143 & 57.4 & \\
\hline \multicolumn{6}{|l|}{ Gender } \\
\hline Female & 83 & 16.4 & 28 & 11.2 & 0.059 \\
\hline Male & 422 & 83.6 & 221 & 88.8 & \\
\hline \multicolumn{6}{|l|}{ Cirrhosis } \\
\hline No & 241 & 47.7 & 119 & 47.8 & 0.986 \\
\hline Yes & 264 & 52.3 & 130 & 52.2 & \\
\hline \multicolumn{6}{|l|}{ ALB (g/dL) } \\
\hline$\leq 3.5$ & 46 & 9.1 & 22 & 8.8 & 0.902 \\
\hline$>3.5$ & 459 & 90.9 & 227 & 91.2 & \\
\hline \multicolumn{6}{|c|}{ TB $(\mu \mathrm{mol} / \mathrm{L})$} \\
\hline$\leq 17$ & 399 & 79.0 & 199 & 79.9 & 0.772 \\
\hline$>17$ & 106 & 21.0 & 50 & 20.1 & \\
\hline \multicolumn{6}{|l|}{ HBsAg } \\
\hline Negative & 67 & 13.3 & 49 & 19.7 & 0.022 \\
\hline Positive & 438 & 86.7 & 200 & 80.3 & \\
\hline \multicolumn{6}{|l|}{$\mathrm{HCV}$} \\
\hline Negative & 438 & 86.7 & 249 & 100 & 0.000 \\
\hline Positive & 67 & 13.3 & 0 & 0 & \\
\hline \multicolumn{6}{|c|}{ AFP (ng/mL) } \\
\hline$\leq 20$ & 249 & 49.3 & 131 & 52.6 & 0.394 \\
\hline$>20$ & 256 & 50.7 & 118 & 47.4 & \\
\hline \multicolumn{6}{|c|}{ Tumor number } \\
\hline Single & 447 & 88.5 & 217 & 87.1 & 0.586 \\
\hline Multiple & 58 & 11.5 & 32 & 12.9 & \\
\hline \multicolumn{6}{|c|}{ Differentiation } \\
\hline$|-| \mid$ & 310 & 61.4 & 159 & 63.9 & 0.511 \\
\hline III-IV & 195 & 38.6 & 90 & 36.1 & \\
\hline \multicolumn{6}{|c|}{ Encapsulation } \\
\hline None & 164 & 32.5 & 84 & 33.7 & 0.592 \\
\hline Yes & 341 & 67.5 & 165 & 66.3 & \\
\hline \multicolumn{6}{|c|}{ Tumor size (cm) } \\
\hline$\leq 5$ & 331 & 65.5 & 160 & 64.3 & 0.727 \\
\hline$>5$ & 174 & 34.5 & 89 & 35.7 & \\
\hline \multicolumn{6}{|c|}{ BCLC stage } \\
\hline $0+A$ & 323 & 64.0 & 157 & 63.1 & 0.038 \\
\hline B & 148 & 29.3 & 62 & 24.9 & \\
\hline C & 34 & 6.7 & 30 & 12.0 & \\
\hline \multicolumn{6}{|c|}{ Chinese stage ${ }^{*}$} \\
\hline la & 282 & 55.8 & 136 & 54.6 & 0.020 \\
\hline lb & 160 & 31.7 & 60 & 24.1 & \\
\hline Ila & 27 & 5.3 & 22 & 8.8 & \\
\hline Illb & 2 & 0.4 & 1 & 0.4 & \\
\hline Illa & 34 & 6.7 & 30 & 12.0 & \\
\hline \multicolumn{6}{|c|}{ Vascular invasion } \\
\hline No & 297 & 58.8 & 135 & 54.2 & 0.045 \\
\hline Micro & 174 & 34.5 & 84 & 33.7 & \\
\hline Macro & 34 & 6.7 & 30 & 12.0 & \\
\hline
\end{tabular}

*, Guidelines for Diagnosis and Treatment of Primary Liver Cancer in China (2017 Edition). Liver Cancer 2018;7:23560. HCC, hepatocellular carcinoma; HBsAg, hepatitis B surface antigen; ALB, albumin; AFP, $\alpha$-fetoprotein; TB, total bilirubin. 
Table S2 Concentrations of serum PON1 and AFP in different groups (sub groups) in both test and validation cohorts

\begin{tabular}{|c|c|c|c|c|c|c|c|}
\hline Variable & Group & \multicolumn{3}{|c|}{ Test } & \multicolumn{3}{|c|}{ Validation } \\
\hline \multicolumn{8}{|c|}{ PON1 (ng/mL) } \\
\hline \multirow[t]{2}{*}{ Total } & NVI & 297 & $346.4(211.0-525.7)$ & 416.9 & 135 & 505.1 (346.0-614.9) & 660.1 \\
\hline & MVI & 174 & 124.5 (38.4-262.8) & 185.6 & 84 & 160.9 (103.9-188.2) & 178.3 \\
\hline \multirow[t]{3}{*}{$\mathrm{sHCC}$} & $\mathrm{NVI}$ & 228 & $338.6(202.2-521.0)$ & 414.8 & 103 & 509.9 (365.0-614.2) & 702.8 \\
\hline & MVI & 93 & 129.9 (52.1-233.8) & 182.9 & 47 & $163.4(102.8-184.7)$ & 164.7 \\
\hline & GVI & 10 & $32.3(9.8-102.9)$ & 77.0 & 10 & $65.2(0.0-285.6)$ & 138.8 \\
\hline $\mathrm{aHCC}$ & $\mathrm{NVI}$ & 69 & $370.2(235.6-553.4)$ & 423.8 & 32 & $441.3(303.7-623.7)$ & 522.5 \\
\hline \multicolumn{8}{|c|}{ AFP (ng/mL) } \\
\hline \multirow[t]{3}{*}{ Total } & NVI & 297 & $10.3(3.3-113.1)$ & 578.7 & 135 & $15.3(4.3-133.7)$ & 709.6 \\
\hline & MVI & 174 & $41.0(8.0-1,032.2)$ & $3,439.5$ & 84 & $19.8(6.1-521.9)$ & $3,018.9$ \\
\hline & GVI & 34 & $794.8(164.4-4,368.8)$ & $9,166.5$ & 30 & $40.3(10.8-4,245.0)$ & $5,851.1$ \\
\hline \multirow[t]{3}{*}{$\mathrm{sHCC}$} & $\mathrm{NVI}$ & 228 & $9.9(3.4-104.4)$ & 209.4 & 103 & $13.6(3.6-79.0)$ & 386.3 \\
\hline & MVI & 93 & $16.9(4.7-172.8)$ & 401.1 & 47 & $15.3(6.2-345.6)$ & 382.9 \\
\hline & GVI & 10 & $760.4(441.4-1,087.8)$ & 726.6 & 10 & $13.4(9.4-64.6)$ & $6,500.2$ \\
\hline $\mathrm{aHCC}$ & NVI & 69 & 11.7 (3.3-124.7) & $1,798.9$ & 32 & $56.5(11.0-1,414.2)$ & $1,750.3$ \\
\hline
\end{tabular}

HCC, hepatocellular carcinoma; NVI, no vascular invasion; MVI, micro-vascular invasion; GVI, gross vascular invasion; AFP, $\alpha$-fetoprotein; PON1, paraoxonase 1; aHCC, advanced-HCC. 
Table S3 Supplementary data of the ROC curves in different groups in both test and validation cohorts

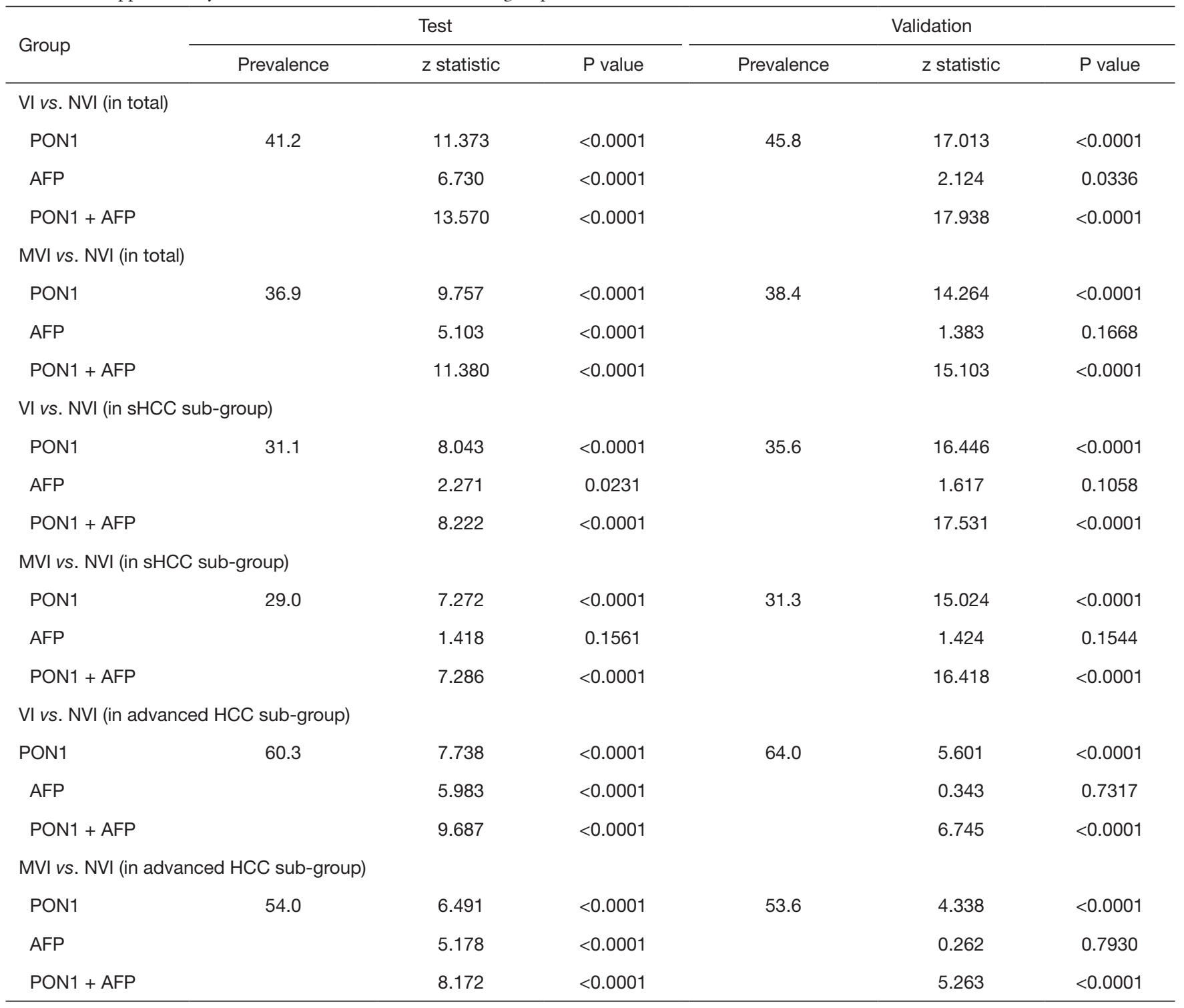

VI, vascular invasion; NVI, no vascular invasion; MVI, micro-vascular invasion; AFP, $\alpha$-fetoprotein; PON1, paraoxonase 1. 